\title{
MicroRNA-let-7f-1 is induced by lycopene and inhibits cell proliferation and triggers apoptosis in prostate cancer
}

\author{
DANBIN LI ${ }^{1}$, LINA CHEN ${ }^{2}$, WENRAN ZHAO ${ }^{3}$, JIANBING HAO $^{4}$ and RUIHUA AN ${ }^{1}$ \\ ${ }^{1}$ Department of Urological Surgery, The First Affiliated Hospital of Harbin Medical University, Harbin, \\ Heilongjiang 150001; ${ }^{2}$ Department of Biophysics, College of Bioinformatics Science and Technology; \\ ${ }^{3}$ Department of Cell Biology, Harbin Medical University, Harbin, Heilongjiang 150081; \\ ${ }^{4}$ Department of Nephrology, The First Affiliated Hospital of Harbin Medical University, \\ Harbin, Heilongjiang 150001, P.R. China
}

Received February 26, 2015; Accepted December 21, 2015

DOI: $10.3892 / \mathrm{mmr} .2016 .4841$

\begin{abstract}
Previous studies have suggested that lycopene has cytotoxic effects in a variety of types of human cancer. An improved understanding of the mechanisms underlying the anticancer effects of lycopene may provide novel therapeutic targets for cancer treatment. PC3 cells were treated with different concentrations of lycopene for 24 and $48 \mathrm{~h}$, the level of protein kinase B (AKT2) was detected by reverse transcription-quantitative polymerase chain reaction (RT-qPCR) and western blotting. Additionally, the expression levels of microRNA (miR)-let-7f-1 were measured using RT-qPCR. miR-let-7f-1 function was analyzed using cell proliferation and apoptosis assays in gain- and loss-of-function experiments. It was observed that lycopene downregulated the expression of AKT2 and upregulated the expression of miR-let-7f-1 in PC3 cells. Re-introduction of miR-let-7f-1 into PC3 cells was able to inhibit cell proliferation and induce apoptosis. Further investigation indicated that miR-let-7f-1 targeted AKT2 in PC3 cells and upregulation of AKT2 could attenuate the effects induced by miR-let-7f-1. The results of the current study indicate that miR-let-7f-1 is involved in the anticancer effects of lycopene and serves an important role in the inhibition of prostate cancer progression through the downregulation of AKT2.
\end{abstract}

\section{Introduction}

Prostate cancer is the most common cancer diagnosed and the second leading cause of cancer-associated mortality in males in the USA, with an estimated 220,800 newly diagnosed cases and 27,540 cases of cancer-associated mortality

Correspondence to: Professor Ruihua An, Department of Urological Surgery, The First Affiliated Hospital of Harbin Medical University, 23 Youzheng Street, Harbin, Heilongjiang 150001, P.R. China

E-mail: anruihuahydyy@163.com

Key words: miR-let-7f-1, lycopene, prostate cancer, AKT2, apoptosis predicted to occur in 2015 in the USA (1). Lycopene, a carotenoid found in tomatoes, exhibits multiple bioactivities and has been reported to protect against prostate cancer via inhibition of cancer cell proliferation and induction of apoptotic cell death (2-5). A previous study suggested that lycopene regulates the breast cancer cell cycle and apoptosis via the suppression of expression of cell cycle regulatory proteins (6). Additional studies have reported that high intakes of tomato-based products were associated with a $10-20 \%$ reduction in the risk of prostate cancer $(7,8)$. However, the molecular mechanisms responsible for the effects of lycopene on prostate cancer remain to be fully elucidated.

MicroRNAs (miRNAs) are a class of endogenous small non-coding RNAs that regulate the expression of target genes at transcriptional and post-transcriptional levels $(9,10)$. Previous studies have reported that miRNAs may function as oncogenes or tumor suppressors in various types of cancer, including lung cancer, breast cancer, hepatocellular carcinoma and gastric cancer (11-14). Additionally, studies have observed aberrant expression of numerous miRNAs in prostate cancer (15-17). In addition, circulating miRNAs in serum or plasma samples have been demonstrated to be associated with patient survival, and may be developed as potential biomarkers for prostate cancer diagnosis and recurrence (18). Let-7f-1 was reported to inhibit proliferation, migration and in vivo tumor formation of human glioblastoma cancer cells by downregulating the expression of the oncogenes pan-RAS, N-RAS and K-RAS (19). A previous study demonstrated that miR-let-7f-1 mediated cisplatin resistance via targeting secreted protein, acidic, cysteine-rich (osteonectin) (SPARC), a crucial regulator of multiple cellular signal transduction pathways (20). Let-7f was reported to be upregulated in primary breast cancer using miRNA microarray, however was not validated by reverse transcription-quantitative polymerase chain reaction (RT-qPCR) analysis (21). Previous studies have indicated that lycopene inhibited prostate cancer progression though multiple growth factor-mediated signaling pathways (22-25). Therefore, whether specific miRNAs targeting growth factor signalling pathways are involved in the antitumor activity of lycopene remains to be further elucidated. 


\section{Materials and methods}

Cell culture and treatment. Human prostate carcinoma cells PC3 (CRL-1435) were purchased from the American Type Culture Collection (Manassas, VA, USA) and were maintained in RPMI 1640 media (Invitrogen; Thermo Fisher Scientific, Inc., Waltham, MA, USA) supplemented with $10 \%$ fetal bovine serum (FBS; Invitrogen; Thermo Fisher Scientific, Inc.) at $37^{\circ} \mathrm{C}$ in a $5 \% \mathrm{CO}_{2}$ incubator. Lycopene (Sigma-Aldrich, St. Louis, MO, USA) was dissolved in tetrahydrofuran (Sigma-Aldrich) as $20 \mathrm{mM}$ stock solution and maintained at $-20^{\circ} \mathrm{C}$. For the experiments, $\mathrm{PC} 3$ cells were cultured in serial concentrations of lycopene $(10,20$ and $50 \mu \mathrm{m})$ and control cultures were treated with water only.

$R N A$ extraction and RT-qPCR. Total RNA was isolated from cells using TRIzol ${ }^{\circledR}$ reagent (Invitrogen; Thermo Fisher Scientific, Inc.) according to the manufacturer's instructions. For miR-let-7f-1 detection, miRNA was polyadenylated and reverse transcribed into cDNA with the One Step PrimeScript miRNA cDNA Synthesis kit (Takara Biotechnology, Co., Ltd., Dalian, China) in triplicate. For AKT2 detection, cDNA (50 ng) was synthesized using the PrimeScript RT reagent kit (Takara Biotechnology, Co., Ltd.) according to the manufacturer's protocol. RT-qPCR analysis was performed with SYBR Green (Takara Biotechnology, Co., Ltd.) in an ABI 7500 Fast Real-Time PCR System (Applied Biosystems; Thermo Fisher Scientific, Inc.). The small nuclear RNA U6 and glyceraldehyde 3-phosphate dehydrogenase (GAPDH) mRNA were used as internal controls for miRNA and mRNA detection, respectively. The forward primers for the miR-let-7f-1 and U6 were synthesized by Biosune Co., Ltd. (Shanghai, China), and the sequences were as follows: miR-let-7f-1, 5' CTATACAATCTATTGCCTTCCC 3' and U6, 5' TGCGGGTGCTCGCTTCGGCAGC 3'. The reverse primers for the miR-let-7f-1 and U6 were universal adaptor primers available in a ready-to-go format in the One Step PrimeScript miRNA cDNA Synthesis kit (D350A; Takara Biotechnology, Co., Ltd). The primers for the AKT2 and GAPDH genes were obtained from Primerbank (http://pga.mgh.harvard.edu/primerbank/) and the sequences were as follows: AKT2, forward 5'-AGG CACGGGCTAAAGTGAC-3' and reverse 5'-CTGTGTGAG CGACTTCATCCT-3'; and GAPDH, forward 5'-CTGGGCTAC ACTGAGCACC-3' and reverse 5'-AAGTGGTCGTTGAGG GCAATG-3'. The $\Delta \Delta \mathrm{Cq}$ method (26) was used in the analysis of the PCR data.

Oligonucleotide transfection. miR-let-7f-1 mimics, miR-let-7f-1 inhibitors (anti-miR-let-7f-1) and their corresponding controls, scramble and negative controls (NC), respectively, were synthesized by Shanghai GenePharma Co. Ltd. (Shanghai, China). Cells were transfected with $50 \mathrm{nM}$ oligonucleotides using Superfect ${ }^{\mathrm{TM}}$ Transfection Reagent (Qiagen, Inc., Valencia, CA, USA) according to the manufacturer's instructions. Subsequent to $48 \mathrm{~h}$ transfection, cells were harvested and the miR-let-7f-1 expression level was confirmed by RT-qPCR.

Cell proliferation assay and apoptosis assay. Following $24 \mathrm{~h}$ cultivation, cells were transfected with $50 \mu \mathrm{m}$ lycopene. To measure the effect of miR-let-7f-1 mimics or lycopene on cell proliferation, cells $\left(2 \times 10^{3}\right)$ were incubated in 96-well plates in $100 \mu \mathrm{l}$ medium containing $10 \%$ FBS. A total of $10 \mu \mathrm{l}$ WST-1 (Roche Diagnostics GmbH, Mannheim, Germany) was added to each well at the indicated time points and the plate was incubated for another $2 \mathrm{~h}$ at $37^{\circ} \mathrm{C}$. The absorbance was measured at $450 \mathrm{~nm}$ using a microtiter plate reader (Spectra Rainbow; Tecan Group Ltd., Männedorf, Switzerland) according to the manufacturer's instructions. Relative optical density was calculated using a Spectra Rainbow microplate reader (Tecan Group, Ltd., Männedorf, Switzerland) in three independent experiments.

Cell apoptosis assay was determined using the Annexin V-Fluorescein Isothiocyanate (FITC) Apoptosis Detection kit (BD Pharmingen, San Diego, CA, USA) according to the manufacturer's instructions. PC3 cells treated as indicated were harvested and resuspended in $100 \mu \mathrm{l}$ annexin V-FITC labeling solution containing $5 \mu \mathrm{l}$ annexin V-FITC and $5 \mu \mathrm{l}$ propidium iodide (BD Pharmingen) and incubated for $30 \mathrm{~min}$ at room temperature in dark. Subsequent to incubation, the samples were analyzed by the FC500 Flow Cytometer (Beckman Coulter, Inc., Miami, FL, USA). Each measurement was performed in quadruplicate and each experiment was repeated at least three times.

Western blotting. Whole cell protein lysates were extracted using radioimmunoprecipitation assay buffer (Thermo Fisher Scientific, Inc.) containing $200 \mathrm{ml} \mathrm{Na}_{3} \mathrm{VO}_{4}, 200 \mathrm{mM}$ $\mathrm{NaF}, 0.5 \mathrm{M}$ ethylenediaminetetraacetic acid and proteinase inhibitors, for $30 \mathrm{~min}$ on ice. The protein concentrations were quantified with Bio-Rad protein assay reagent (Bio-Rad Laboratories, Inc., Hercules, CA, USA). Equal amounts of proteins $(40 \mu \mathrm{g})$ were separated by $10 \%$ sodium dodecyl sulfate-polyacrylimide gel electrophoresis (Bio-Rad Laboratories, Inc.) and transferred onto polyvinylidene difluoride membranes (EMD Millipore, Billerica, MA, USA), which were incubated with primary monoclonal antibodies against rabbit AKT2 $(1: 1,000 ; 2964)$, mouse phosphorylated AKT2 (Ser474) (1:1,000; 12694; both Cell Signaling Technology, Inc., Danvers, MA, USA) and rabbit GAPDH (1:1,000; sc-47724; Santa Cruz Biotechnology, Inc., Santa Cruz, CA, USA) at $4^{\circ} \mathrm{C}$ overnight. The membranes were washed 3 times in Tris-buffered saline with Tween-20 (TBST) and incubated with the corresponding horseradish peroxidase-conjugated secondary bovine anti-mouse (sc-2371)and anti-rabbit (sc-2370) IgG antibodies, (both 1:1,000, Santa Cruz Biotechnology, Inc.) for $1 \mathrm{~h}$ at room temperature. Subsequent to three washes with TBST, the bound secondary antibody was detected using an enhanced chemiluminescence system (Pierce Biotechnology, Inc., Rockford, IL, USA). The signals were detected using a SuperSignal Protein Detection kit (Pierce Biotechnology, Inc.). The band density of specific proteins was quantified subsequent to normalization with the density of GAPDH using ImageJ (version 1.48; National Institutes of Health, Bethesda, MA, USA.

Bioinformatics and luciferase assay. In silico analyses were performed to determine the putative miRNAs able to target AKT2. TargetScan 6.2 software (http://www.targetscan.org) was used and the 3 'untranslated region (UTR) target regions 
A
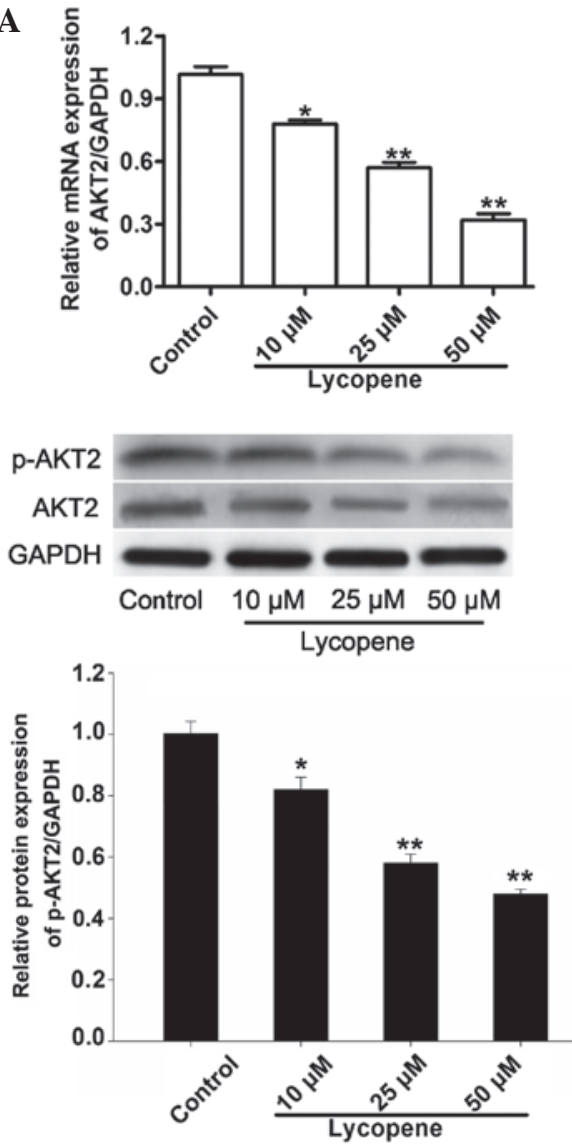

B
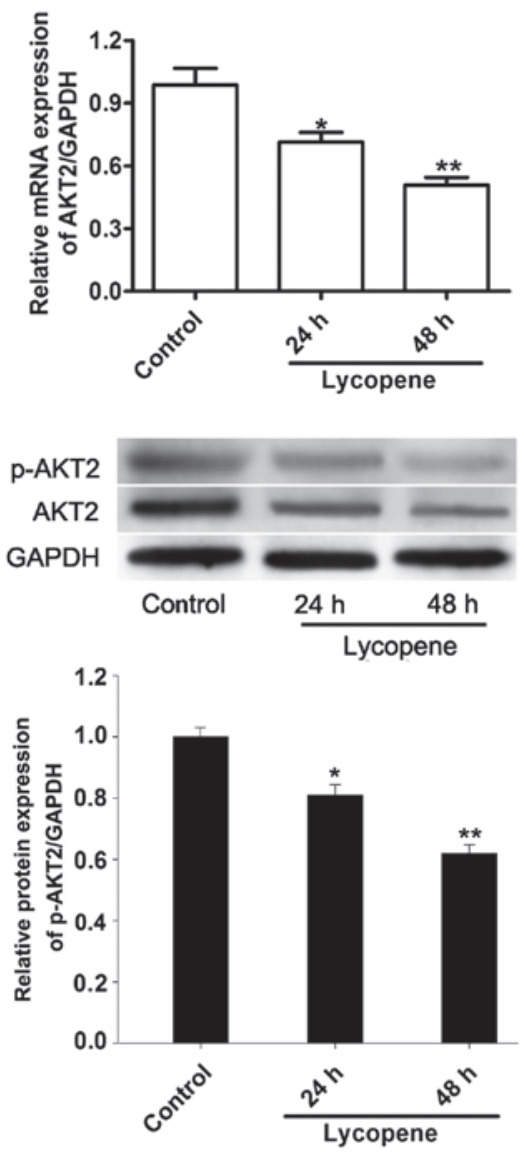

Figure 1. Lycopene reduced the expression of AKT2 in PC3 cells. (A) PC3 cells were treated with different doses of lycopene as indicated for 48 h, then the mRNA expression levels of AKT2 were evaluated by RT-qPCR (upper panel) and the protein levels of AKT2 were evaluated by western blotting (lower panel). (B) PC3 cells were treated with $25 \mu \mathrm{M}$ lycopene for 24 and $48 \mathrm{~h}$, then the mRNA expression levels of AKT2 were evaluated by RT-qPCR and the protein levels of AKT2 were evaluated by western blotting. GAPDH was used as the loading control. ${ }^{*} \mathrm{P}<0.05$, ${ }^{* *} \mathrm{P}<0.01$. RT-qPCR, reverse transcription-quantitative polymerase chain reaction; GAPDH, glyceraldehyde 3-phosphate dehydrogenase; p-AKT2, phosphorylated-AKT2.

were selected in order to determine miRNA recognition elements which were involved in cell proliferation.

The fulllength 3'UTR of the AKT2 genewasPCR-amplified from genomic DNA and inserted into the XhoI and NotI sites of the psi-CHECK2 vector (Promega Corporation, Madison, WI, USA), downstream of the luciferase gene, to generate the plasmids AKT2-UTR-WT. The sequences of primers used were: Forward 5'-AAACTCGAGGCAGTCTGCCCACGC AGA-3' and reverse 5'-AAAGCGGCCGCCAGGACTGCT GGTAGCACCA-3'. AKT2-UTR-MUT plasmids were generated from AKT2-UTR-WT by mutating the miRNA binding site using a Quick Change Site-Directed Mutagenesis kit (Stratagene; Agilent Technologies, Inc., Santa Clara, CA, USA) with the following primers: 5'-TGGGCACAGGCC TGGCGGGGTCATCTTTTTAGTGCCTCTC-3' (forward) and 5'-GAGAGGCACTAAAAAGATGACCCCGCCAGG CCTGTGCCCA-3' (reverse). All constructs were verified by sequencing.

For the luciferase reporter assay, $\mathrm{PC} 3$ cells were cultured in 96 -well plates and incubated at $37^{\circ} \mathrm{C}$ for $24 \mathrm{~h}$ prior to transfection. Luciferase reporter constructs and miR-let-7f-1 mimics or miR-let-7f-1 inhibitors were transfected using Superfect ${ }^{\mathrm{TM}}$ transfection reagent (Qiagen, Inc.). Luciferase activity was measured using the Dual Luciferase Reporter Assay System (Promega Corporation) $48 \mathrm{~h}$ post-transfection.
Renilla luciferase activity was normalized to firefly luciferase activity. All transfection experiments were conducted in triplicate and repeated 3 times independently.

Statistical analysis. Data were expressed as the mean \pm standard deviation of at least three independent experiments. Statistical analyses were analyzed using Student's two-tailed t-test. All analyses were performed with SPSS software, version 15.0 (SPSS, Inc., Chicago, IL, USA). P<0.05 was considered to indicate a statistically significant difference.

\section{Results}

Lycopene downregulated the expression of AKT2. Previous reports have demonstrated that lycopene was able to inhibit the growth of human colon cancer cells and breast cancer cells by altering PI3K/Akt signaling pathways (27-29), which served a central role in the promotion of cell proliferation and the inhibition of cell death $(30,31)$. We investigated whether the expression of AKT2 was reduced by lycopene. As indicated in Fig. 1, lycopene reduced the expression of AKT2 in a time- and dose-dependent manner at mRNA and protein levels. The mRNA levels of AKT2 in the PC3 cells were reduced by 19,42 and $67 \%$ in response to treatment with 10,25 and $50 \mu \mathrm{M}$ lycopene at $48 \mathrm{~h}$ compared with the 
A
AKT2-UTR
MUT 5 ' -UGGGCACAGGCCUGGCGGGGUCA-3'
WT $5^{\prime}$-UGGGCACAGGCCUGGCUACCUCA-3'
| | | | ||| ||

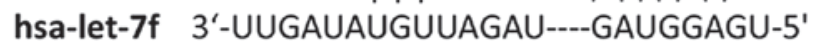

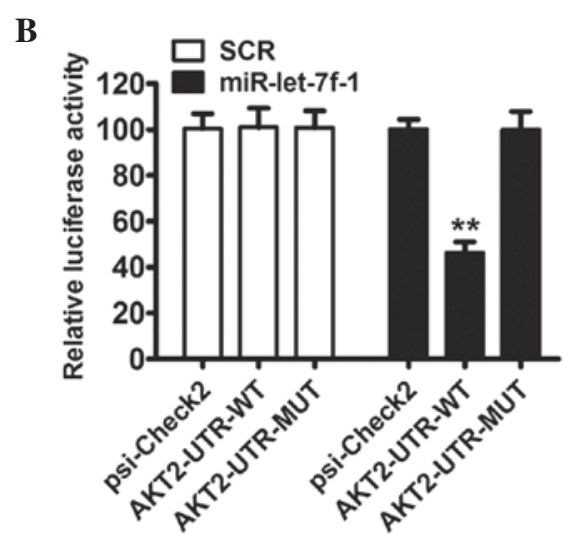

E
C

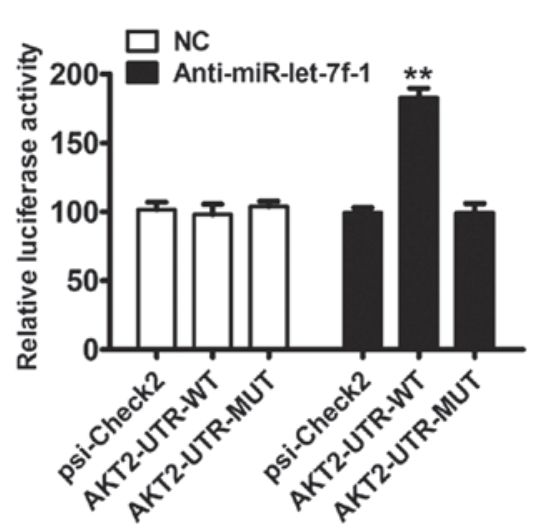

D

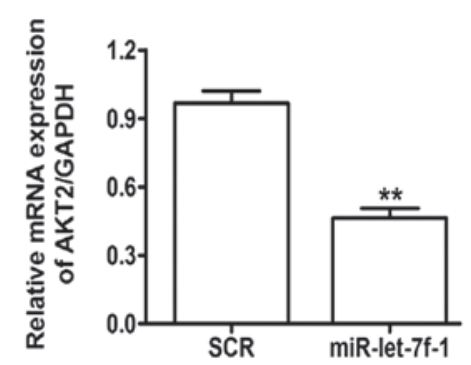

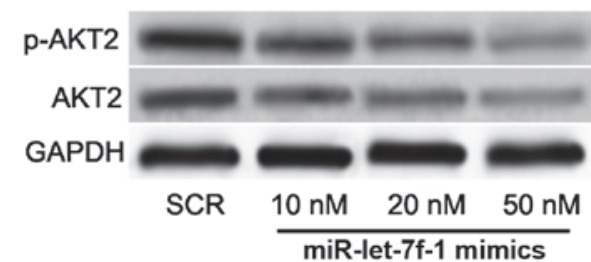

$\mathbf{F}$

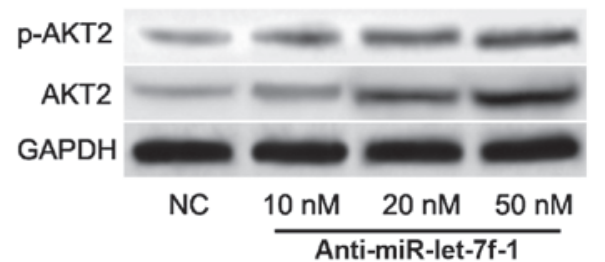

Figure 2. AKT2 was identified as target gene of miR-let-7f-1. (A) A schematic representation indicating the putative target site for miR-let-7f-1 in the AKT2 mRNA 3'UTR. (B) Luciferase assays on PC3 cells transfected with the psi-CHECK2 control, AKT2-UTR-WT or AKT2-UTR-MUT reporters and miR-let-7f-1 mimic oligonucleotides or SCR. (C) Luciferase assays on PC3 cells transfected with the psi-CHECK2 control, AKT2-UTR-WT or AKT2-UTR-MUT reporters and miR-let-7f-1 inhibitor oligonucleotides or NC. (D) The relative mRNA expression levels of AKT2 were determined by reverse transcription-quantitative polymerase chain reaction. (E) Endogenous protein levels of AKT2 and phosphorylated AKT2 in PC3 cells subsequent to transfection with miR-let-7f-1 and scramble control oligonucleotides were detected by western blotting. (F) Western blot assay indicating the expression levels of AKT2 and p-AKT2 in PC3 cells following transfection with the miR-let-7f-1 inhibitor and NC. ${ }^{* *} \mathrm{P}<0.01$. miR, microRNA; UTR, untranslated region; WT, wild-type; MUT, mutant; NC, negative control; p-AKT2, phosphorylated AKT2; GAPDH, glyceraldehyde 3-phosphate dehydrogenase; SCR, scramble.

control (Fig. 1A, upper panel). Meanwhile the protein levels of AKT2 and phosphorylated AKT2 in the PC3 cells were significantly reduced by 18,42 and $52 \%$ following treatment with 10,25 , and $50 \mu \mathrm{M}$ lycopene at $48 \mathrm{~h}$ (Fig. 1A, lower panel). Treatment of PC 3 cells with $25 \mu \mathrm{M}$ lycopene for $24 \mathrm{~h}$ or $48 \mathrm{~h}$ also significantly reduced AKT2 mRNA expression levels by 29 and $49 \%$ (Fig. 1B, upper panel) and protein levels by 19 and 38\% (Fig. 1B, lower panel).

miR-let-7f-1 regulates AKT2 expression through direct binding to its $3^{\prime} U T R$. It is known that that miRNAs exert their biological functions by binding to the 3'-UTR and inhibiting expression of their target genes (9). For this reason, bioinformatics were used to predict the potential miRNAs targeting AKT2. By searching TargetScan, AKT2 was identified as the target of miR-let-7f-1 with the highest possibility.

To confirm the negative regulation of miR-let-7f-1 on AKT2, the 3'-UTR of AKT2 was cloned into a luciferase reporter construct (Fig. 2A). The reporter assay indicated that overexpression of miR-let-7f-1 triggered a marked reduction of luciferase activity of the AKT2-UTR-WT plasmid by 54\% in PC3 cells compared with the scramble control, without alteration in luciferase activity of AKT2-UTR-MUT (Fig. 2B). By contrast, inhibition of miR-let-7f-1 dramatically led to a marked increase of luciferase activity of AKT2-UTR-WT by $91 \%$, without alterations in luciferase activity of AKT2-UTR-MUT (Fig. 2C). Consistent with the reporter assay, a significant reduction of AKT2 mRNA by $50 \%$ was observed in miR-let-7f-1-overexpressed cells compared with the scramble control in PC3 cells (Fig. 2D). Furthermore, western blot analysis demonstrated that overexpression of miR-let-7f-1 following administration of 10, 20 and $50 \mathrm{nM}$ miR-let-7f-1 mimics was able to downregulate the expression of AKT2 and phosphorylated AKT2 by 35, 45 and $66 \%$, respectively (Fig. 2E). miR-let-7f-1 knockdown at 10, 25 and $50 \mathrm{~nm}$ anti-miR-let-7f-1 treatment resulted in a marked increase in AKT2 and phosphorylated AKT2 expression by 42,69 and $88 \%$, respectively, as compared with the NC group (Fig. 2F). These data indicate that AKT2 is likely to be regulated by miR-let-7f-1 in prostate cancer at transcriptional and post-transcriptional levels.

miR-let-7f-1 inhibited the proliferative abilities and induced apoptosis in vitro. Previous studies have demonstrated that miR-let-7f-1 was involved in the suppression of cell proliferation $(19,20)$, thus it is hypothesized that the expression of miR-let-7f-1 is augmented by lycopene in PC 3 cells. In order to test whether lycopene induced the upregulation of miR-let-7f-1, 

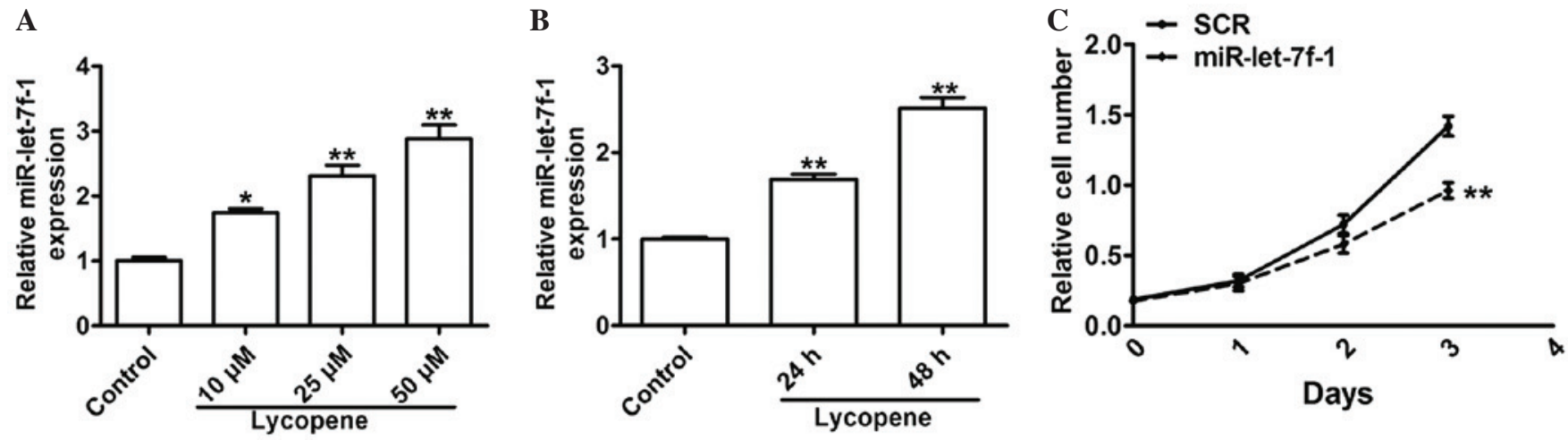

D

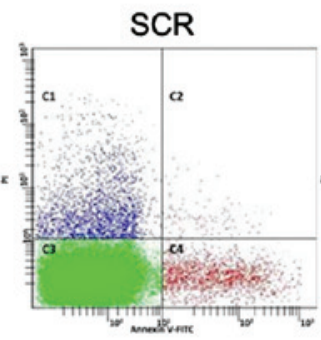

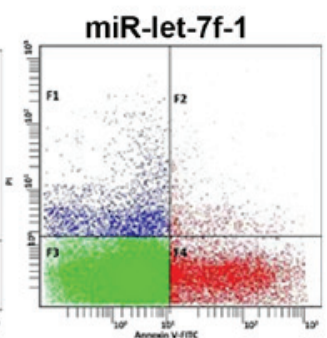

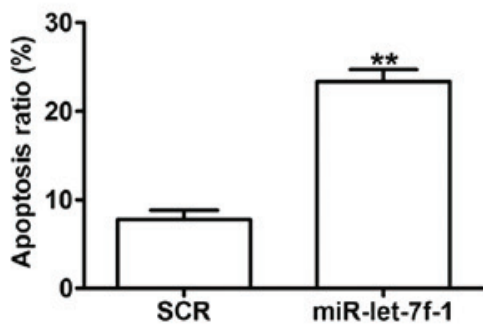

Figure 3. Effect of miR-let-7f-1 on growth and apoptosis of PC3 cells. (A) The relative expression of miR-let-7f-1 was determined by RT-qPCR in PC3 cells pretreated with lycopene $(10,25$ and $50 \mu \mathrm{M})$ for $48 \mathrm{~h}$. (B) RT-qPCR was used to determine the expression of miR-let-7f-1 in PC 3 cells pretreated with $25 \mu \mathrm{M}$ lycopene for 24 or $48 \mathrm{~h}$. (C) The growth rate of the cells was detected using the WST-1 assay. (D) Flow cytometry analysis of apoptosis in PC3 cells transfected with SCR or miR-let-7f-1. "P<0.05; ;* $\mathrm{P}<0.01$. miR, microRNA; RT-qPCR, reverse transcription-quantitative polymerase chain reaction; SCR, scramble.

A

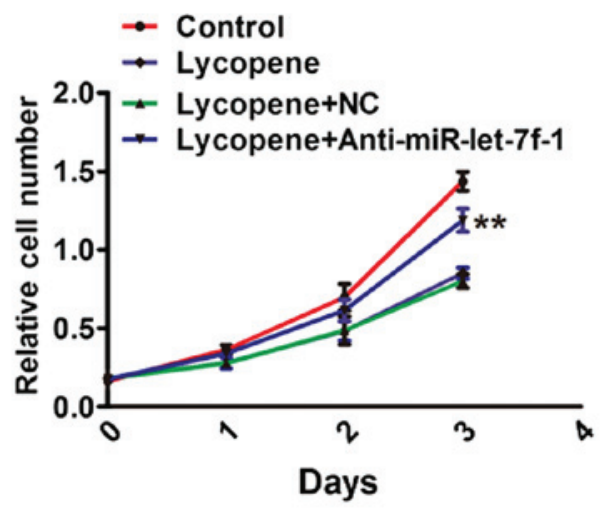

C

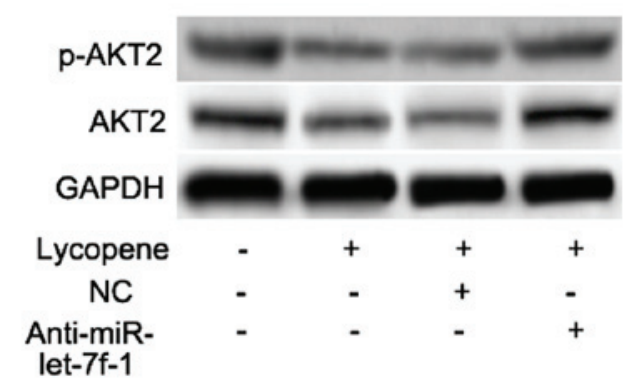

B
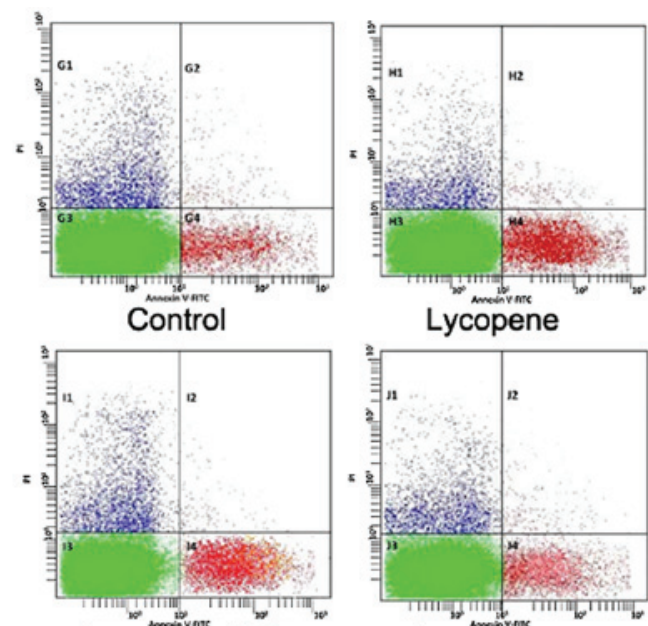

Lycopene+NC

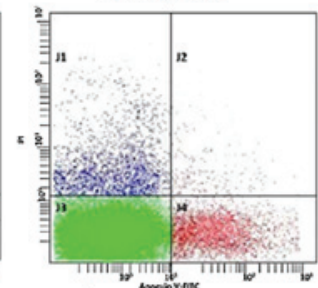

Lycopene+

Anti-miR-Let-7f-1

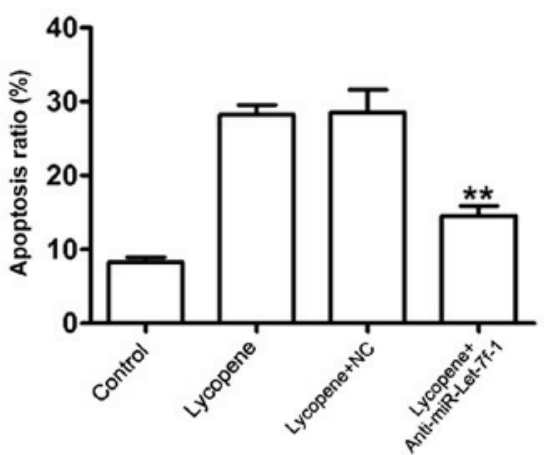

Figure 4. Knockdown of miR-let-7f-1 partly rescued lycopene-dependent suppression of cell functions and AKT2 expression. (A) PC3 cells were treated as indicated and were measured by the WST-1 assay at different time periods. (B) Apoptotic cells were determined by fluorescence-activated cell sorting. (C) Western blot analysis of AKT2 and p-AKT2 protein expression in PC 3 cells treated as indicated. GAPDH was regarded as the endogenous control. ${ }^{* *} \mathrm{P}<0.01$ vs. NC. miR, microRNA; NC, negative control; p-AKT2, phosphorylated AKT2; GAPDH, glyceraldehyde 3-phosphate dehydrogenase. 
miR-let-7f-1 expression was detected by RT-qPCR in PC3 cells treated with lycopene. The RT-qPCR assay indicated that the expression of miR-let-7f-1 was enhanced by 74,131 and $188 \%$ in response to lycopene treatment at 10,25 and $50 \mu \mathrm{M}$, in a dose-dependent manner (Fig. 3A). A significant increase of 69 and $151 \%$ in miR-let-7f-1 expression was also observed in response to $25 \mu \mathrm{m}$ lycopene treatment at 24 and $48 \mathrm{~h}$, in a time-dependent manner (Fig. 3B). In order to investigate the role of miR-let-7f-1 in prostate cancer cells, miR-let-7f-1 mimics were transfected into PC3 cells. A proliferation assay was performed to evaluate cell growth and the data demonstrated that the increased expression of miR-let-7f-1 induced significant inhibition on cell proliferation by 7, 20 and $32 \%$ at days 1, 2 and 3, respectively (Fig. 3C). Apoptosis assays demonstrated that PC3 cells that were transfected with miR-let-7f-1 mimics exhibited an increase in the apoptotic rate by $200 \%$, as compared with those of the scramble controls (Fig. 3D). These data indicated that miR-let-7f-1 induced by lycopene may effectively inhibit the growth and enhance apoptotic levels of PC3 cells in vitro.

Anti-miR-let-7f-1 oligonucleotides partly reversed the downregulation of AKT2 induced by lycopene in prostate cancer PC 3 cells. To further confirm that lycopene downregulated the expression of AKT2 by upregulation of miR-let-7f-1, PC 3 cells were treated $50 \mu \mathrm{M}$ lycopene, followed by transfection with $50 \mathrm{nM}$ anti-miR-let-7f-1 oligonucleotides for $48 \mathrm{~h}$. Using the WST-1 assay, it was demonstrated that anti-miR-let-7f-1 attenuated the inhibition of cell proliferation caused by lycopene in PC 3 cells by 18,21 and $28 \%$ by days 1, 2 and 3, respectively (Fig. 4A). In addition, knockdown of miR-let-7f-1 in PC3 cells incubated with lycopene resulted in significantly reduced cell apoptosis (47\%; Fig. 4B), indicating reversal of the increased effects of lycopene on PC3 cell apoptosis. In addition, anti-miR-let-7f-1 oligonucleotides partly abolished the inhibitory effect of lycopene on AKT2 and phosphorylated AKT2 protein expression (96\%; Fig. 4C). Thus, it was confirmed that miR-let-7f-1 was a key mediator of growth inhibition and apoptotic enhancement of lycopene in prostate cancer.

\section{Discussion}

Lycopene, which is naturally present in tomato carotenoids, has been suggested to exhibit potential anticancer activity in several types of human cancer, including prostate, colon and breast cancer $(4,5,27)$. In addition, lycopene has been reported to inhibit the development of certain cases of chemically induced carcinogenesis $(32,33)$. Epidemiological and clinical studies have suggested that increased consumption of tomato products and greater blood concentrations of lycopene are associated with a reduced risk of prostate cancer $(34,35)$. However, the inhibitory effect and possible molecular mechanisms of lycopene, including cell proliferation arrest and/or apoptosis induction, remain poorly understood.

In the current study, a novel molecular mechanism of lycopene in cancer control was proposed. For the first time, to the best of our knowledge, it was demonstrated that lycopene may inhibit cellular proliferation progression and induce apoptosis in PC3 cells, via upregulating miR-let-7f-1 expression and inhibiting the expression of AKT2. It has been previously reported that AKT2, an important member of the PI3K signaling pathway, is activated in prostate cancer $(30,36)$. Subsequently, the expression of AKT2 was investigated. Upon treatment with lycopene, a time- and dose-dependent reduction in AKT2 expression was observed, with the greatest inhibition observed when PC3 cells were treated with $50 \mu \mathrm{m}$ for $48 \mathrm{~h}$, indicating that the growth-inhibitory activity of lycopene likely reduced the mRNA and protein levels of AKT2. However, the mechanism by which lycopene downregulates AKT2 expression remains unclear. Previous studies have indicated that miRNAs act as fine-tuning regulators of protein expression $(9,10)$. With an aim to identify miRNAs regulating AKT2 expression in prostate cancer, it was identified that miR-let-7f-1 could significantly downregulate AKT2 expression, which was supported by the luciferase reporter assay.

Therefore, it is notable to examine whether miR-let-7f-1 had an effect on cell proliferation and cell apoptosis. It was identified that the upregulation of miR-let-7f-1 was able to significantly inhibit the growth of PC3 cells. Upregulation of miR-let-7f-1 could also enhance apoptosis of PC 3 cells. To the best of our knowledge, the current study was the first to investigate the roles and possible mechanisms of miR-let-7f-1 upregulation in PC3 cells induced by lycopene. In addition, it remains unclear whether miR-let-7f- 1 is required to reduce the level of phosphorylated Akt in PC3 cells pretreated with lycopene. The data of the current study indicated that knockdown of miR-let-7f-1 could partly reverse the inhibitory effects of lycopene on cell proliferation and AKT2 expression in PC3 cells. In addition, lycopene-induced apoptotic induction was abrogated by knockdown of miR-let-7f-1 in PC3 cells. Taken together, it is suggested that lycopene-mediated growth inhibition and apoptosis is mediated through downregulating AKT2 and at both the mRNA and protein levels.

In conclusion, the data of the current study indicate that lycopene downregulates AKT2 expression via an miRNA pathway. These observations suggest that lycopene may be a potential anticancer compound with therapeutic applications.

\section{References}

1. Siegel RL, Miller KD and Jemal A: Cancer Statistics, 2015. CA Cancer J Clin 65: 5-29, 2015.

2. Zu K, Mucci L, Rosner BA, Clinton SK, Loda M, Stampfer MJ and Giovannucci E: Dietary lycopene, angiogenesis and prostate cancer: A prospective study in the prostate-specific antigen era. J Natl Cancer Inst 106: djt430, 2014.

3. Wei MY and Giovannucci EL: Lycopene, tomato products and prostate cancer incidence: A review and reassessment in the PSA screening era. J Oncol 2012: 271063, 2012.

4. Kristal AR, Till C, Platz EA, Song X, King IB, Neuhouser ML, Ambrosone CB and Thompson IM: Serum lycopene concentration and prostate cancer risk: Results from the prostate cancer prevention trial. Cancer Epidemiol Biomarkers Prev 20: 638-646, 2011.

5. Teodoro AJ, Oliveira FL, Martins NB, Maia GA, Martucci RB and Borojevic R: Effect of lycopene on cell viability and cell cycle progression in human cancer cell lines. Cancer Cell Int 12: 36, 2012.

6. Hwang ES and Bowen PE: Cell cycle arrest and induction of apoptosis by lycopene in LNCaP human prostate cancer cells. J Med Food 7: 284-289, 2004.

7. Venkateswaran V and Klotz LH: Diet and prostate cancer: Mechanisms of action and implications for chemoprevention. Nat Rev Urol 7: 442-453, 2010.

8. Syed DN, Suh Y, Afaq F and Mukhtar H: Dietary agents for chemoprevention of prostate cancer. Cancer Lett 265: 167-176, 2008. 
9. Bartel DP: MicroRNAs: Target recognition and regulatory functions. Cell 136: 215-233, 2009.

10. Ventura A and Jacks T: MicroRNAs and cancer: Short RNAs go a long way. Cell 136: 586-591, 2009.

11. Yanaihara N, Caplen N, Bowman E, Seike M, Kumamoto K, Yi M, Stephens RM, Okamoto A, Yokota J, Tanaka T, et al: Unique microRNA molecular profiles in lung cancer diagnosis and prognosis. Cancer Cell 9: 189-198, 2006.

12. Iorio MV, Ferracin M, Liu CG, Veronese A, Spizzo R, Sabbioni S, Magri E, Pedriali M, Fabbri M, Campiglio M, et al: MicroRNA gene expression deregulation in human breast cancer. Cancer Res 65: 7065-7070, 2005.

13. Zhu XC, Dong QZ, Zhang XF, Deng B, Jia HL, Ye QH, Qin LX and $\mathrm{Wu} X Z$ : microRNA-29a suppresses cell proliferation by targeting SPARC in hepatocellular carcinoma. Int J Mol Med 30 $1321-1326,2012$

14. Huang B, Luo W, Sun L, Zhang Q, Jiang L, Chang J, Qiu X and Wang E: MiRNA-125a-3p is a negative regulator of the RhoA-actomyosin pathway in A549 cells. Int J Oncol 42: 1734-1742, 2013.

15. Coppola V, De Maria R and Bonci D: MicroRNAs and prostate cancer. Endocr Relat Cancer 17: F1-F17, 2010.

16. Rajendiran S, Parwani AV, Hare RJ, Dasgupta S, Roby RK and Vishwanatha JK: MicroRNA-940 suppresses prostate cancer migration and invasion by regulating MIEN1. Mol Cancer 13: 250, 2014.

17. Goto Y, Kurozumi A, Enokida H, Ichikawa T and Seki N: Functional significance of aberrantly expressed microRNAs in prostate cancer. Int J Urol 22: 242-252, 2015.

18. Jackson BL, Grabowska A and Ratan HL: MicroRNA in prostate cancer: Functional importance and potential as circulating biomarkers. BMC Cancer 14: 930, 2014

19. Dong Q, Meng P, Wang T, Qin W, Qin W, Wang F, Yuan J, Chen Z, Yang A and Wang H: MicroRNA let-7a inhibits proliferation of human prostate cancer cells in vitro and in vivo by targeting E2F2 and CCND2. PLoS One 5: e10147, 2010.

20. Pannuru P, Dontula R, Khan AA, Herbert E, Ozer H, Chetty C and Lakka SS: miR-let-7f-1 regulates SPARC mediated cisplatin resistance in medulloblastoma cells. Cell Signal 26: 2193-2201, 2014.

21. Yan LX, Huang XF, Shao Q, Huang MY, Deng L, Wu QL, Zeng YX and Shao JY: MicroRNA miR-21 overexpression in human breast cancer is associated with advanced clinical stage, lymph node metastasis and patient poor prognosis. RNA 14: 2348-2360, 2008

22. Trejo-Solís C, Pedraza-Chaverrí J, Torres-Ramos M, Jiménez-Farfán D, Cruz Salgado A, Serrano-García N, Osorio-Rico L and Sotelo J: Multiple molecular and cellular mechanisms of action of lycopene in cancer inhibition. Evid Based Complement Alternat Med 2013: 705121, 2013.

23. Tang Y, Parmakhtiar B, Simoneau AR, Xie J, Fruehauf J, Lilly $\mathrm{M}$ and $\mathrm{Zi} \mathrm{X}$ : Lycopene enhances docetaxel's effect in castration-resistant prostate cancer associated with insulin-like growth factor I receptor levels. Neoplasia 13: 108-119, 2011

24. Lo HM, Hung CF, Tseng YL, Chen BH, Jian JS and Wu WB: Lycopene binds PDGF-BB and inhibits PDGFBB-induced intracellular signaling transduction pathway in rat smooth muscle cells. Biochemical Pharmacology 74: 54-63, 2007.
25. Chen ML, Lin YH, Yang CM and Hu ML: Lycopene inhibits angiogenesis both in vitro and in vivo by inhibiting MMP-2/uPA system through VEGFR2-mediated PI3K-AKT and ERK/p38 signaling pathways. Molecular Nutrition \& Food Research 56: 889-899, 2012

26. Livak and Schmittgen: Analysis of relative gene expression data using real-time quantitative PCR and the $2-\Delta \Delta \mathrm{Ct}$ method. Methods 25: 402-408, 2001.

27. Tang FY, Shih CJ, Cheng LH, Ho HJ and Chen HJ: Lycopene inhibits growth of human colon cancer cells via suppression of the Akt signaling pathway. Mol Nutr Food Res 52: 646-654, 2008.

28. Takeshima M, Ono M, Higuchi T, Chen C, Hara T and Nakano S: Anti-proliferative and apoptosis-inducing activity of lycopene against three subtypes of human breast cancer cell lines. Cancer Sci 105: 252-257, 2014.

29. Palozza P, Colangelo M, Simone R, Catalano A, Boninsegna A, Lanza P, Monego G and Ranelletti FO: Lycopene induces cell growth inhibition by altering mevalonate pathway and Ras signaling in cancer cell lines. Carcinogenesis 31: 1813-1821, 2010.

30. Carver BS, Chapinski C, Wongvipat J, Hieronymus H, Chen Y, Chandarlapaty S, Arora VK, Le C, Koutcher J, Scher H, et al: Reciprocal feedback regulation of PI3K and androgen receptor signaling in PTEN-deficient prostate cancer. Cancer Cell 19: 575-586, 2011.

31. Hsu AL, Ching TT, Wang DS, Song X, Rangnekar VM and Chen CS: The cyclooxygenase-2 inhibitor celecoxib induces apoptosis by blocking Akt activation in human prostate cancer cells independently of Bcl-2. J Biol Chem 275: 11397-11403, 2000.

32. Kim DJ, Takasuka N, Kim JM, Sekine K, Ota T, Asamoto M, Murakoshi M, Nishino H, Nir Z and Tsuda H: Chemoprevention by lycopene of mouse lung neoplasia after combined initiation treatment with DEN, MNU and DMH. Cancer Lett 120: 15-22, 1997.

33. Narisawa T, Fukaura Y, Hasebe M, Nomura S, Oshima S, Sakamoto H, Inakuma T, Ishiguro Y, Takayasu J and Nishino H: Prevention of N-methylnitrosourea-induced colon carcinogenesis in F344 rats by lycopene and tomato juice rich in lycopene. Jpn J Cancer Res 89: 1003-1008, 1998.

34. Giovannucci E: A review of epidemiologic studies of tomatoes, lycopene and prostate cancer. Exp Biol Med (Maywood) 227: 852-859, 2002

35. Pohar KS, Gong MC, Bahnson R, Miller EC and Clinton SK: Tomatoes, lycopene and prostate cancer: A clinician's guide for counseling those at risk for prostate cancer. World J Urol 21: 9-14, 2003

36. Zhong H, Chiles K, Feldser D, Laughner E, Hanrahan C, Georgescu MM, Simons JW and Semenza GL: Modulation of hypoxia-inducible factor 1alpha expression by the epidermal growth factor/phosphatidylinositol 3-kinase/PTEN/AKT/FRAP pathway in human prostate cancer cells: Implications for tumor angiogenesis and therapeutics. Cancer Res 60: 1541-1545, 2000. 